\title{
NO-ARBITRAGE IN HEATH-JARROW-MORTON MODEL AND THE BOND PRICING EQUATION
}

\author{
Man M. Chawla \\ X-027, Regency Park II, DLF City Phase IV \\ Gurgaon-122002, Haryana, INDIA
}

\begin{abstract}
In the present paper we discuss the relationship of no-arbitrage in Heath-Jarrow-Morton (H-J-M) model and no-arbitrage in the bond pricing partial differential equation approach. We show that the no-arbitrage condition of H-J-M translates, in terms of zero-coupon bonds, into the bond pricing equation. Conversely, we show that affine-yield solutions of the bond pricing equation, for the very general four-parameter short rate model, satisfy the HJ-M no-arbitrage condition without actually obtaining the solutions.
\end{abstract}

AMS Subject Classification: 91B24, 91B28, 91B30

Key Words: forward rate, Heath-Jarrow-Morton model, short rate models, no-arbitrage, bond pricing equation

\section{Introduction}

A wide class of interest rate models that have been proposed is based on the evolution of the short rate. These models often lead to a partial differential equation for pricing of zero-coupon bonds, the so-called bond pricing equation which admits simple affine-yield solutions. No-arbitrage is ensured by equating return from the constructed portfolio of bonds with different maturities to the corresponding return from a risk-free deposit. By construction, though these models match the short rate with the yield at the initial point, it is generally difficult to calibrate such a model to incorporate the whole initial yield curve into the model. 
On the other hand, the Heath-Jarrow-Morton (H-J-M) model, in terms of forward rate, is based on the evolution of the whole yield curve. The noarbitrage condition in H-J-M specifies drift in the risk-neutral dynamics of the forward rate in terms of a certain function of the volatility.

In the present paper we discuss the relationship of no-arbitrage in H-J-M model and no-arbitrage in the bond pricing partial differential equation approach. We show that the no-arbitrage condition of H-J-M translates, in terms of zero-coupon bonds, into the bond pricing equation. We then show affine-yield solutions of the bond pricing equation satisfy the no-arbitrage condition of $\mathrm{H}$ J-M. This holds, in particular, for the well-known short rate models of Vasicek [10] and Cox, Ingersoll and Ross (C-I-R) [2]. In fact, conversely we show that affine-yield solutions of the bond pricing equation, for the very general fourparameter short rate model, satisfy the H-J-M no-arbitrage condition without actually obtaining the solutions. This general four-parameter short rate model (see, for example, Wilmott et al. [11]) includes the models of Vasicek and C-I$\mathrm{R}$, thus obviating the need to obtain solutions for each model individually and then verifying for the no-arbitrage condition of H-J-M.

The H-J-M model was developed by Heath, Jarrow and Morton in [7]. An easy-to-understand text book account of H-J-M model is given in Shreve [9]. Related works are Heath, Jarrow and Morton [5], [6]. Filipov [3] and Jara [8] consider H-J-M type models in terms of interest rate futures.

We collect here some basic preliminary concepts needed for discussion in subsequent sections.

Let $B(r, t, T)$ (or, simply $B(t, T)$ or $B(r, t)$ depending on context) denote the price of a zero-coupon bond at time $t$ with maturity value one at time $T$, $t \leq T$. Forward rate at time $t$ for a future time $T, T \geq t$, is defined as

$$
f(t, T)=-\frac{\partial}{\partial T} \ln B(t, T) .
$$

Note that $f(t, T)$ is also a function of $r$; in fact, $f(t, T)$ is short for $f(r, t, T)$. Bond prices can be recovered by integrating (1.1),

$$
\int_{t}^{T} f(t, v) d v=-\int_{t}^{T} \frac{\partial}{\partial v} \ln B(t, v) d v=-\ln B(t, T),
$$

since $B(t, t)=1$. Therefore,

$$
B(t, T)=e^{-\int_{t}^{T} f(t, v) d v} .
$$

Alternatively, this can be written as

$$
B(t, T)=e^{-(T-t) Y(t, T)},
$$


where we have set

$$
Y(t, T)=\frac{1}{T-t} \int_{t}^{T} f(t, v) d v .
$$

$Y(t, T)$ is the yield between times $t \rightarrow T$. Plot of $Y$ versus $T-t$ is the yield curve. With (1.2) we can express the yield (1.5) as

$$
Y(t, T)=-\frac{1}{T-t} \ln B(t, T) .
$$

Again, with (1.1) we can write (1.6) as

$$
f(t, T)=\frac{\partial}{\partial T}[Y(t, T)(T-t)],
$$

which is the interest rate implied by the yield curve, also written as $r(t, T)$. Thus,

$$
f(t, T)=r(t, T)=r(T),
$$

for short. Note that instantaneous rate at $t$,

$$
f(t, t)=r(t),
$$

is the short rate at $t$. Note also that setting $T=t$ in (1.6) gives

$$
\left.Y(t, T)\right|_{T=t}=-\left.\frac{\partial}{\partial T} B(t, T)\right|_{T=t} .
$$

But from (1.3) we have

$$
\left.\frac{\partial}{\partial T} B(t, T)\right|_{T=t}=-f(t, t)=-r(t) .
$$

Therefore,

$$
\left.Y(t, T)\right|_{T=t}=r(t) .
$$

So,

$$
f(t, t)=Y(t, t)=r(t, t)=r(t) .
$$

Now, in the notation of (1.8) bond price in (1.3) can be written as

$$
B(t, T)=e^{-\int_{t}^{T} r(v) d v},
$$

and $e^{-\int_{t}^{T} r(v) d v}$ is the discount factor between times $t \rightarrow T$. In particular, the discount factor between times $0 \rightarrow t$ is

$$
D(t)=e^{-\int_{0}^{t} r(v) d v} .
$$


Finally, it is interesting to know relationship of the interest rate implied by the yield curve $r(T)$ with the yield curve itself. For the purpose, for fixed $t$ we rewrite $(1.7)$ as

$$
r(T)=\frac{d}{d \tau}[Y(\tau) \tau]=Y(\tau)+\tau \frac{d Y}{d \tau},
$$

where we have set time to expiry $\tau=T-t$. Now, consider the tangent line to the yield curve at $\tau$ :

$$
\ell(s)=Y(\tau)+(s-\tau) \frac{d Y}{d \tau} .
$$

In view of $(1.12)$, from (1.13) we obtain

$$
r(T)=\left.\ell(s)\right|_{s=2 \tau} .
$$

Thus, $r(T)$ is the point on the line $\ell(s)$ tangent to the yield curve for $s=2 \tau$.

\section{No-Arbitrage in H-J-M Model}

In this section we introduce the H-J-M model and derive the no-arbitrage condition which expresses drift in the risk-neutral dynamics of the forward rate as a certain function of volatility.

Assume that the initial forward rate curve $f(0, T)$ is known at time 0 . In the H-J-M model, forward rate at time $t, 0 \leq t \leq T$, evolves as

$$
f(t, T)=f(0, T)+\int_{0}^{t} \phi(u, T) d u+\int_{0}^{t} \psi(u, T) d W(u),
$$

or in differential form ( $d$ is differential with respect to $t$ with $T$ held fixed),

$$
d f(t, T)=\phi(t, T) d t+\psi(t, T) d W(t) .
$$

Here, $W(t)$ is Brownian motion under actual measure $\mathbb{P}$, and $\phi(t, T)$ is the drift of $f(t, T)$ under actual measure. For fixed $T, \phi(t, T)$ and $\psi(t, T)$ are adapted processes in the variable $t$.

To find dynamics of discounted bond price in H-J-M model, since

$$
d\left(-\int_{t}^{T} f(t, v) d v\right)=f(t, t) d t-\int_{t}^{T} d f(t, v) d v
$$

with (2.2) from (2.3) we have

$$
d\left(-\int_{t}^{T} f(t, v) d v\right)=\left\{r(t)-\phi^{*}(t, T)\right\} d t-\psi^{*}(t, T) d W(t)
$$


where we have set

$$
\phi^{*}(t, T)=\int_{t}^{T} \phi(t, v) d v, \psi^{*}(t, T)=\int_{t}^{T} \psi(t, v) d v .
$$

Therefore, by Ito-Doeblin formula, from (1.3) by writing $B(t, T)=e^{x}$ we obtain (henceforth dropping the arguments, unless needed explicitly),

$$
d B=B d\left(-\int_{t}^{T} f(t, v) d v\right)+\frac{1}{2} B\left(d\left(-\int_{t}^{T} f(t, v) d v\right)\right)^{2},
$$

and with (2.4) we get

$$
d B=B\left[r-\phi^{*}+\frac{1}{2} \psi^{* 2}\right] d t-B \psi^{*} d W .
$$

Now, with the discount factor $D(t)$, the differential of the discounted bond price is given, with (2.6), by

$$
\begin{aligned}
d(D(t) B(t, T)) & =-D r B d t+D d B \\
& =-D B\left[\left\{\phi^{*}-\frac{1}{2} \psi^{* 2}\right\} d t+\psi^{*} d W\right] .
\end{aligned}
$$

Set

$$
\phi^{*}-\frac{1}{2} \psi^{* 2}=\psi^{*} \theta(t)
$$

then (2.7) becomes

$$
\begin{aligned}
d(D(t) B(t, T)) & =-\psi^{*} D B[\theta(t) d t+d W(t)] \\
& =-\psi^{*} D B d \widetilde{W}(t),
\end{aligned}
$$

where we have set

$$
\widetilde{W}(t)=\int_{0}^{t} \theta(u) d u+W(t) .
$$

Using Girsanov's theorem [4] we can now change to a probability measure $\widetilde{\mathbb{P}}$ under which $\widetilde{W}(t)$ is a Brownian motion and $(2.9)$ means that $D(t) B(t, T)$ is a martingale under risk-neutral probability measure $\widetilde{\mathbb{P}}$.

Finally, for the H-J-M model under risk-neutral measure, from (2.6) we can write 5

$$
\begin{aligned}
d B(t, T) & =B\left[r-\psi^{*} \theta\right] d t-B \psi^{*} d W(t) \\
& =B r d t-B \psi^{*} d \widetilde{W}(t) .
\end{aligned}
$$


Differentiating (2.8) with respect to $T$ we have

$$
\phi-\psi \psi^{*}=\psi \theta(t)
$$

therefore from (2.2) we get

$$
\begin{aligned}
d f(t, T) & =\phi d t+\psi d W(t) \\
& =\left[\psi \psi^{*}+\psi \theta(t)\right] d t+\psi d W(t) \\
& =\psi \psi^{*} d t+\psi d \widetilde{W}(t) .
\end{aligned}
$$

Thus, the no-arbitrage condition in H-J-M model is

$$
\text { drift of } f(t, T)=\psi(t, T) \psi^{*}(t, T) \text {. }
$$

\section{H-J-M No-Arbitrage Condition and the Bond Pricing Equation}

In this section we show that the no-arbitrage condition in H-J-M leads to the partial differential equation, called the bond pricing equation, for the determination of the price of zero-coupon bonds.

Consider the short rate modelled by the stochastic differential equation:

$$
d r(t)=u(t, r(t)) d t+w(t, r(t)) d W(t),
$$

where $d W(t)$ is a Brownian process under actual measure $\mathbb{P}$. The dynamics of bond prices driven by the forward rate (2.2) is given by (2.6) which can now be written as

$$
d B-r B d t=-\psi^{*} B\left[\left\{\frac{\phi^{*}-\frac{1}{2} \psi^{* 2}}{\psi^{*}}\right\} d t+d W\right]
$$

The condition of no-arbitrage in the H-J-M model is the existence of a $\theta(t)$ as given in (2.8):

$$
\theta(t)=\frac{\phi^{*}-\frac{1}{2} \psi^{* 2}}{\psi^{*}}
$$

and (3.2) can be written as

$$
d B-r B d t=-\psi^{*} B[\theta(t) d t+d W(t)] .
$$


Here $\theta(t)$ is the market price of risk for zero-coupon bonds. Again, with $B(r, t, T)$ considered as a function of $r$ and $t$, for fixed $T$, with dynamics of short rate (3.1) by Ito's formula we get

$$
\begin{aligned}
d B(r, t) & =B_{t} d t+B_{r} d r+\frac{1}{2} B_{r r}(d r)^{2} \\
& =B_{t} d t+B_{r}[u d t+w d W]+\frac{1}{2} w^{2} B_{r r} d t \\
& =\left(B_{t}+u B_{r}+\frac{1}{2} w^{2} B_{r r}\right) d t+w B_{r} d W
\end{aligned}
$$

Therefore,

$$
\begin{aligned}
d B-r B d t & =\left[-r B+B_{t}+u B_{r}+\frac{1}{2} w^{2} B_{r r}\right] d t+w B_{r} d W \\
& =\left(w B_{r}\right)\left[\left(\frac{-r B+B_{t}+u B_{r}+\frac{1}{2} w^{2} B_{r r}}{w B_{r}}\right) d t+d W\right] \\
& =\left(w B_{r}\right)[\lambda d t+d W],
\end{aligned}
$$

where we have set

$$
\lambda(r, t)=\frac{-r B+B_{t}+u B_{r}+\frac{1}{2} w^{2} B_{r r}}{w B_{r}} .
$$

In (3.5) $\lambda$ represents market price of risk. Comparing (3.4) and (3.5) we have

$$
\lambda(r(t), t)=\theta(t) \text { and }-\psi^{*} B=w B_{r} .
$$

Equation (3.6) can be rewritten as

$$
-r B+B_{t}+u B_{r}+\frac{1}{2} w^{2} B_{r r}=\lambda w B_{r},
$$

or equivalently, as

$$
B_{t}+\frac{1}{2} w^{2} B_{r r}+(u-\lambda w) B_{r}-r B=0 .
$$

This is the zero-coupon bond pricing equation for the short rate driven by (3.1).

Motivated by the coefficient of $B_{r}$ in (3.8), let us now specify the coefficient of $d t$ in (3.1) as

$$
u(r, t)=v(r, t)+\lambda(r, t) w(r, t) .
$$


Then (3.1) becomes

$$
\begin{aligned}
d r & =v d t+w[\lambda d t+d W] \\
& =v d t+w d \widetilde{W}
\end{aligned}
$$

by Girsanov's theorem where $d \widetilde{W}$ represents Brownian motion under risk-neutral measure $\widetilde{\mathbb{P}}$. Now, from (1.1) dynamics of the forward rate, regarding $B(r, t)$ as a function of $r$ and $t$, is given by

$$
d f(t, T)=-\frac{\partial}{\partial T}\left(\frac{1}{B} d B\right)=-\frac{\partial}{\partial T}\left[\frac{1}{B}\left\{B_{t} d t+B_{r} d r\right\}\right]
$$

With (3.10) this becomes

$$
\begin{aligned}
d f(t, T) & =-\frac{\partial}{\partial T}\left[\frac{1}{B}\left\{B_{t} d t+B_{r}(v d t+w d \widetilde{W})\right\}\right] \\
& =\left[-\frac{\partial}{\partial T}\left(\frac{B_{t}+v B_{r}}{B}\right)\right] d t+\left[-\frac{\partial}{\partial T}\left(w \frac{B_{r}}{B}\right)\right] d \widetilde{W}
\end{aligned}
$$

This is an H-J-M model with $\psi=-\frac{\partial}{\partial T}\left(w \frac{B_{r}}{B}\right)$. Since we are working under risk-neutral measure, the H-J-M no-arbitrage condition (2.14) now applied to $(3.11)$ is

$$
\begin{aligned}
-\frac{\partial}{\partial T}\left(\frac{B_{t}+v B_{r}}{B}\right) & =\left\{-\frac{\partial}{\partial T}\left(w \frac{B_{r}}{B}\right)\right\}\left\{\int_{t}^{T}-\frac{\partial}{\partial u}\left(w \frac{B_{r}}{B}(r, t, u)\right) d u\right\} \\
& =\left\{-\frac{\partial}{\partial T}\left(w \frac{B_{r}}{B}\right)\right\}\left\{-\left.\left(w \frac{B_{r}}{B}(r, t, u)\right)\right|_{u=t} ^{u=T}\right\} .
\end{aligned}
$$

Since $B(r, t, t)=1$ and $B_{r}(r, t, t)=0$, this condition works out to

$$
\begin{aligned}
-\frac{\partial}{\partial T}\left(\frac{B_{t}+v B_{r}}{B}\right) & =\left\{-\frac{\partial}{\partial T}\left(w \frac{B_{r}}{B}\right)\right\}\left\{-w \frac{B_{r}}{B}\right\} \\
& =\frac{\partial}{\partial T}\left\{\frac{1}{2} w^{2}\left(\frac{B_{r}}{B}\right)^{2}\right\} .
\end{aligned}
$$

Now, integrating (3.12) with respect to $T$ we have

$$
\frac{B_{t}+v B_{r}}{B}+\frac{1}{2} w^{2}\left(\frac{B_{r}}{B}\right)^{2}=a(r(t), t),
$$


for some function $a$ of $r(t)$ and possibly $t$. Set

$$
\left(B_{r}\right)^{2}=B B_{r r},
$$

then (3.13) becomes

$$
B_{t}+v B_{r}+\frac{1}{2} w^{2} B_{r r}=a(r, t) B
$$

From (1.3) with (2.3), we have

$$
B_{t}=B \frac{d}{d t}\left(-\int_{t}^{T} f(t, u) d u\right)=B\left[f(t, t)-\int_{t}^{T} \frac{d}{d t} f(t, u) d u\right] .
$$

Since $f(t, t)=r(t)$, setting $T=t$ in the above we have $\left.B_{t}\right|_{T=t}=r(t)$. Again, since $B(t, t)=1$, setting $T=t$ in (3.15) we get

$$
a(r, t)=r(t),
$$

and (3.15) becomes

$$
B_{t}+\frac{1}{2} w^{2} B_{r r}+v B_{r}-r B=0 .
$$

Thus, the H-J-M no-arbitrage condition in the present case becomes (3.16) which is the zero-coupon bond pricing equation without reference to the market price of risk.

The condition (3.14) works out to

$$
\frac{B_{r r}}{B_{r}}=\frac{B_{r}}{B}
$$

Integrating with respect to $r$,

$$
\ln B_{r}=\ln B+\ln k,
$$

or equivalently,

$$
\frac{B_{r}}{B}=k=-C(t, T),
$$

where $k=-C(t, T)$ is independent of $r$. Integrating again with respect to $r$,

$$
\ln B=-r C(t, T)+A(t, T),
$$

where $A((t, T)$ is also independent of $r$. This means that

$$
B(t, T)=e^{A(t, T)-r C(t, T)},
$$


implying that only solutions of the bond pricing equation (3.16) possible are of the affine-yield type. In view of the affine-yield solutions possible of the bond pricing equation (3.16), it is reasonable to let the functional forms of $v$ and $w$ to be

$$
v(r, t)=\eta(t)-\gamma(t) r, w(r, t)=\sqrt{\alpha(t) r-\beta(t)} .
$$

We can now express the drift and volatility terms for the forward rate in (2.13) in terms of bond prices. Comparing (2.13) with (3.11) we have

$$
\psi=-w \frac{\partial}{\partial T}\left(\frac{B_{r}}{B}\right),
$$

and together with (3.12) the drift in (2.13) is

$$
\psi \psi^{*}=\frac{1}{2} w^{2} \frac{\partial}{\partial T}\left(\frac{B_{r}}{B}\right)^{2} .
$$

We can also express $\phi$ and $\psi$ individually in terms of bond prices. While $\psi$ is already given by (3.19) in terms of bond prices, we now express $\phi$ in terms of bond prices. Since $\theta(t)$ is independent of $T, \frac{\partial \theta}{\partial T}=0$ and from (3.3) we get

$$
\frac{\partial}{\partial T}\left(\frac{\phi^{*}}{\psi^{*}}\right)=\frac{1}{2} \frac{\partial}{\partial T}\left(\psi^{*}\right) .
$$

Integrating from $t \rightarrow T$ :

$$
\frac{\phi^{*}}{\psi^{*}}=\left.\frac{\phi^{*}(t, T)}{\psi^{*}(t, T)}\right|_{T=t}+\frac{1}{2} \psi^{*} .
$$

By L'Hospital's rule,

$$
\left.\frac{\phi^{*}(t, T)}{\psi^{*}(t, T)}\right|_{T=t}=\frac{\phi(t, t)}{\psi(t, t)},
$$

and therefore,

$$
\phi^{*}=\frac{1}{2} \psi^{* 2}+\frac{\phi(t, t)}{\psi(t, t)} \psi^{*} .
$$

Differentiating (3.21) with respect to $T$, we get

$$
\phi(t, T)=\psi \psi^{*}+\frac{\phi(t, t)}{\psi(t, t)} \psi .
$$


To find $\phi(t, t)$ and $\psi(t, t)$, first note from (3.19) that

$$
\psi(t, T)=-w \frac{\partial}{\partial T}\left(\frac{\partial}{\partial r} \ln B\right)=w \frac{\partial}{\partial r}\left(-\frac{\partial}{\partial T} \ln B\right),
$$

and by (1.1),

$$
\psi(t, T)=w \frac{\partial}{\partial r} f(t, T) .
$$

Set $T=t$, since $f(t, t)=r$, we have

$$
\psi(t, t)=w .
$$

Next, comparing (3.21) with (3.3), since $\lambda=\theta$, we get

$$
\phi(t, t)=\lambda \psi(t, t),
$$

and with (3.23) we have

$$
\phi(t, t)=\lambda w=u-v .
$$

Finally, with (3.20) from (3.22) we have

$$
\phi(t, T)=\frac{1}{2} w^{2} \frac{\partial}{\partial T}\left(\frac{B_{r}}{B}\right)^{2}+\frac{u-v}{w} \psi(t, T) .
$$

As a check, with (3.24) and (3.25) from (2.2) we have

$$
\begin{aligned}
\left.d f(t, T)\right|_{T=t} & =\phi(t, t) d t+\psi(t, t) d W(t) \\
& =\lambda w d t+w d W(t)=w d \widetilde{W}
\end{aligned}
$$

which agrees with (2.13) for $T=t$. Also note that the dynamics of the forward rate (2.2) can be expressed in terms of zero-coupon bonds. From (3.19) and (3.26) we obtain

$$
\begin{aligned}
d f(t, T) & =\left[\frac{1}{2} w^{2} \frac{\partial}{\partial T}\left(\frac{B_{r}}{B}\right)^{2}+\frac{u-v}{w} \psi(t, T)\right] d t+\psi(t, T) d W(t) \\
& =\frac{1}{2} w^{2} \frac{\partial}{\partial T}\left(\frac{B_{r}}{B}\right)^{2} d t-w \frac{\partial}{\partial T}\left(\frac{B r}{B}\right) d \widetilde{W}(t)
\end{aligned}
$$




\section{H-J-M No-Arbitrage Condition in Terms of Solution of the Bond Pricing Equation}

In this section we show affine-yield solutions of the bond pricing equation satisfy the no-arbitrage condition of H-J-M. In fact, we demonstrate this for the very general four-parameter short rate model thus obviating the need to actually obtain these solutions and then verify the no-arbitrage condition of H-J-M.

With the functional forms (3.18) for $v$ and $w$, the zero-coupon bond pricing equation (3.16) becomes

$$
B_{t}+\frac{1}{2}(\alpha r-\beta) B_{r r}+(\eta-\gamma r) B_{r}-r B=0 .
$$

Seeking a solution in the form

$$
B(t, T)=e^{A(t, T)-r C(t, T)},
$$

leads to the following two ordinary differential equations for the determination of $A(t, T)$ and $C(t, T)$ :

$$
\begin{gathered}
A_{t}=\eta C+\frac{1}{2} \beta C^{2}, \\
C_{t}=\frac{1}{2} \alpha C^{2}+\gamma C-1 .
\end{gathered}
$$

Final conditions are $A(T, T)=0$ and $C(T, T)=0$ in order that a bond in (4.2) has a value of one on maturity at $t=T$. Complete discussion of the solutions of bond pricing equations (4.3) and (4.4) is given in Chawla [1]. With (4.2) we now have from (1.1),

$$
f(t, T)=-A_{T}+r C_{T} .
$$

Therefore,

$$
d f=-A_{T}^{\prime} d t+r C_{T}^{\prime} d t+C_{T} d r
$$

where prime denotes differentiation with respect to $t$. With (3.10) we get

$$
d f=\left(-A_{T}^{\prime}+r C_{T}^{\prime}+v C_{T}\right) d t+w C_{T} d \widetilde{W} .
$$

This is an H-J-M model with

$$
\psi=w C_{T} .
$$

Thus, H-J-M no-arbitrage condition becomes

$$
\begin{aligned}
-A_{T}^{\prime}+r C_{T}^{\prime}+v C_{T} & =\left(w C_{T}\right)\left(w \int_{t}^{T} C_{u}(t, u) d u\right) \\
& =w^{2} C C_{T}
\end{aligned}
$$


or equivalently,

$$
\frac{\partial}{\partial T}\left(-A^{\prime}+r C^{\prime}+v C\right)=\frac{1}{2} w^{2} \frac{\partial}{\partial T}\left(C^{2}\right) .
$$

We next show that this no-arbitrage condition of the H-J-M model in terms of the bond pricing equations is satisfied without solving the two ordinary differential equations (4.3) and (4.4) for particular solutions of models like the Varicek and the Cox-Ingersoll-Ross. Integrating (4.5) with respect to $T$ we have

$$
-A^{\prime}+r C^{\prime}+v C=\frac{1}{2} w^{2} C^{2}+m(t),
$$

where $m(t)$ is independent of $T$. Setting $T=t$ we get

$$
\left(-A^{\prime}+r C^{\prime}\right)_{T=t}=m(t)
$$

Since

$$
f(t, T)=\frac{\partial}{\partial T}(-A+r C),
$$

integrating with respect to $T$,

$$
\begin{aligned}
\int_{t}^{T} f(t, s) d s & =\int_{t}^{T} \frac{\partial}{\partial s}(-A(t, s)+r C(t, s)) d s \\
& =-A(t, T)+r C(t, T) .
\end{aligned}
$$

Differentiating with respect to $t$,

$$
-f(t, t)+\int_{t}^{T} \frac{d}{d t} f(t, v) d v=-A^{\prime}+r C^{\prime} .
$$

Setting $T=t$,

$$
-r(t)=\left(-A^{\prime}+r C^{\prime}\right)_{T=t},
$$

and thus in (4.7) $m(t)=-r(t)$. With this (4.6) becomes

$$
-A^{\prime}+r C^{\prime}+v C-\frac{1}{2} w^{2} C^{2}+r(t)=0 .
$$

Substituting for $v$ and $w$ we have

$$
\left(-A^{\prime}+\eta C+\frac{1}{2} \beta C^{2}\right)+r\left(C^{\prime}-\frac{1}{2} \alpha C^{2}-\gamma C+1\right)=0 .
$$

In view of (4.3) and (4.4) this no-arbitrage condition of the H-J-M model is satisfied without any reference to their solutions. 


\section{References}

[1] M.M. Chawla, On solutions of the bond pricing equation, Intern. J. Appl. Math., 23 (2010), 661-680.

[2] J.C. Cox, J.E. Ingersoll and S.A. Ross, A theory of term structure of interest rates, Econometrica, 53 (1985), 385-408.

[3] D. Filipov, Consistence Problems for Heath-Jarrow-Morton Interest Rate Models, Springer, Berlin, 2001.

[4] I.V. Girsanov, On transforming a certain class of stochastic processes by absolutely continuous substitution of measures, Theory Prob. Appl., 5 (1960), 285-301.

[5] D. Heath, R. Jarrow and A. Morton, Contingent claim valuation with a random evolution of interest rates, Rev. Futures Markets, 9 (1990), 54-76.

[6] D. Heath, R. Jarrow and A. Morton, Bond pricing and the term structure of interest rates, A discrete time approximation, J. Fin. Quant. Anal., 25 (1990), 419-440.

[7] D. Heath, R. Jarrow and A. Morton, Bond pricing and the term structure of interest rates: A new methodology, Econometrica, 60 (1992), 77-105.

[8] D. Jara, An extension of Levy's theorem and applications to financial models based on future prices, Ph.D Dissertation, Dept. of Math. Sciences, Carnegi Mellon Univ., 2000.

[9] S.E. Shreve, Stochastic Calculus for Finance II: Continuous-Time Models, Springer International Edition, Third Indian Reprint, New Delhi, 2014.

[10] O. Vasicek, An equilibrium characterization of the term structure, Journal of Financial Economics, 5 (1977), 177-188.

[11] P. Wilmott, S. Howison and J. Dewynne, The Mathematics of Financial Derivatives: A Student Introduction, Cambridge University Press, Cambridge, 1995. 